\title{
FLUXO SALIVAR E USO DE DROGAS PSICOATIVAS EM IDOSOS
}

Marcos Aparecido Sarria Cabrera*, Arthur Eumann Mesas, luiz Angelo Rossato, Selma Maffei de Andrade

Trabalho realizado no Departamento de Saúde Coletiva e Departamento de Clínica Médica do Centro de Ciências da Saúde da Universidade Estadual de Londrina/PR

\author{
*Correspondência: \\ Rua Montese, 65-A \\ Londrina/PR \\ Cep: $86015-020$ \\ Tel/fax: (43) 3324-3524 \\ marcoscabrera@uol.com.br
}

\section{RESUMO}

Овјетіvo. Analisar a associação de fluxo salivar baixo e o uso de drogas psicoativas entre idosos.

Métodos. Estudo transversal com 267 idosos de 60 a 74 anos, residentes em um bairro na cidade de Londrina/PR. Foram excluídos os idosos com alto grau de dependência funcional e os restritos ao leito. O fluxo salivar abaixo de $0,44 \mathrm{ml} / \mathrm{min}$ (primeiro tercil) foi analisado como variável dependente, e o uso contínuo de drogas psicoativas (antidepressivos, anticonvulsivantes, sedativos, antipsicóticos, hipnóticos ou ansiolíticos) foi considerado como variável independente. A análise multivariada foi realizada considerando a interferência do sexo, da idade e do tabagismo.

Resultados. A maioria dos idosos estudados foi do sexo feminino (80,5\%), com uma média de idade de 66,5 anos. 0 uso de drogas psicoativas foi observado em 31 idosos ( I 1,6\%). O fluxo salivar médio foi de $0,76 \mathrm{~m} / \mathrm{min}$, sendo que nos usuários de drogas psicoativas foi de $0,67 \mathrm{ml} / \mathrm{min}$. Na análise multivariada, a utilização de drogas psicoativas estava associada ao fluxo salivar $<0,44 \mathrm{ml} / \mathrm{min}$, independentemente do sexo, da idade e do tabagismo $(p=0,04)$.

Conclusão. Os resultados mostram que, neste grupo de idosos independentes e não institucionalizados, há uma associação entre 0 uso de drogas psicoativas e o baixo fluxo salivar. Estas conclusões fortalecem a necessidade do uso racional destas drogas, principalmente em indivíduos idosos.

Unitermos: Saliva. Xerostomia. Uso de medicamento. Idoso.

\section{INTRODUÇÃO}

Atualmente, nos deparamos com uma ampla utilização de medicamentos psicoativos pela população idosa, principalmente antidepressivos', sedativos ${ }^{2}$ e hipnóticos ${ }^{3}$.

Apesar da grande utilização, estes medicamentos apresentam alguns efeitos colaterais importantes, notadamente entre indivíduos idosos, como a xerostomia, isto é, sensação de secura excessiva na boca ${ }^{4}$. A sensação de boca seca pode ser explicada, em grande parte, pela diminuição da produção de saliva, decorrente da ação anticolinérgica observada nestes grupos farmacológicos ${ }^{5}$.

A saliva está associada a múltiplas funções em relação ao processo de digestão, como paladar, mastigação, formação do bolo alimentar, digestão enzimática e deglutição ${ }^{6}$, ainda, é essencial para a preservação da saúde bucal e orofaríngea ${ }^{4}$. A diminuiç̧ão do fluxo salivar pode trazer como conseqüência inúmeras alterações referentes à saúde bucal, como as cáries, as doenças periodontais, infecções, disfagia, halitose e dificuldades na estabilidade de próteses dentárias ${ }^{7,8}$.

A diminuição de fluxo salivar, propriamente dita, não está relacionada diretamente ao envelhecimento normal ${ }^{9}$. No entanto, a prevalência de xerostomia aumenta muito com a idade e atinge, aproximadamente, $30 \%$ em pessoas com 65 anos ou mais ${ }^{4}$, podendo estar relacionada a fatores como a presença de doenças sistêmicas e o uso contínuo de medicamentos ${ }^{10}$.

A associação de xerostomia e drogas psicoativas foi bem documentada em estudos com idosos ${ }^{4,11}$. No entanto, a concomitância de xerostomia e fluxo salivar baixo não é identificada sistematicamente pelos autores. Nagler ${ }^{12}$ observou que um terço dos indivíduos que se queixam de xerostomia não apresenta diminuição de fluxo salivar e, em um outro estudo com idosos brasileiros não institucionalizados, foi demonstrado que $75 \%$ dos idosos que se queixavam de boca seca não apresentavam diminuição de fluxo salivar' ${ }^{13}$.

Poucos autores estudaram a relação da utilização de drogas com o fluxo salivar em indivíduos idosos e faltam análises da utilização específica de drogas psicoativas ${ }^{13-15}$. Dessa forma, o presente trabalho visa estudar a relação entre o fluxo salivar e uso de drogas psicoativas em idosos da comunidade, funcionalmente independentes e de baixa morbidade.

\section{Métodos}

Foi realizado um estudo transversal com uma população de indivíduos com idade entre 60 e 74 anos residentes na área de abrangência da equipe do Programa de Saúde da Família da unidade básica de saúde de um conjunto habitacional do município de Londrina/PR. O critério de exclusão utilizado foi a presença de dependência funcional importante, com o indivíduo permanecendo restrito ao leito.

Os dados foram coletados por meio de visita domiciliar por uma equipe composta de dois dentistas e seis alunos dos cursos de Medicina e Enfermagem da Universidade Estadual de Londrina, no período de janeiro a abril de 2005. A visita era composta por uma 


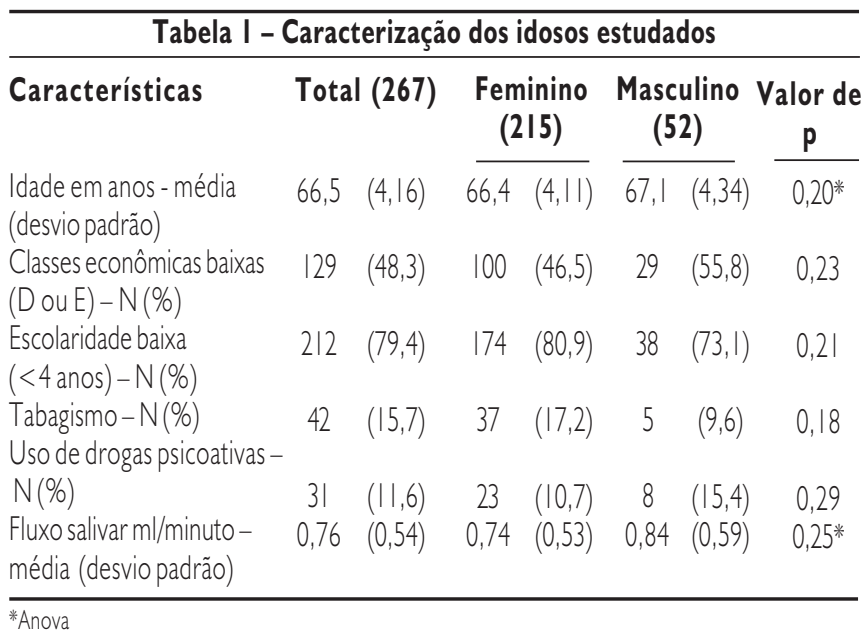

entrevista e um exame clínico odontológico, com medida do fluxo salivar estimulado pela mastigação de tubo de látex natural. A saliva era dispensada a cada 30 segundos e o fluxo foi calculado pelo volume depositado (em $\mathrm{ml}$ ) dividido pelo tempo decorrido (em minutos).

As variáveis analisadas foram:

Variável dependente: fluxo salivar. Foram considerados como indivíduos com fluxo salivar baixo aqueles que apresentassem fluxo inferior ao valor correspondente ao primeiro tercil (fluxo $<0,44 \mathrm{ml} / \mathrm{min}$ ).

Variável independente: uso de drogas psicoativas. Foram considerados os indivíduos que usavam continuamente qualquer tipo de antidepressivos, anticonvulsivantes, sedativos, antipsicóticos, hipnóticos ou ansiolíticos.

Variáveis de controle: idade (em anos), sexo e tabagismo (fumante e não fumante).

Além das variáveis dependente, independente e de controle, foram ainda analisadas a classe econômica, segundo a classificação da Associação Brasileira de Empresas de Pesquisa ${ }^{16}$, e a escolaridade (considerada baixa, se menor que quatro anos). Também foi analisado o tipo de medicamento usado (só na descrição).

Foi realizada uma análise multivariada da associação de baixo fluxo salivar e o uso de drogas psicoativas, considerando a interferência da idade, sexo, tabagismo. O nível se significância considerado foi de $5 \%(p<0,05)$. O programa estatístico utilizado foi o Epi Info, versão $3.3 .2^{17}$.

projeto foi aprovado previamente pelo Comitê de Ética em Pesquisa da Universidade Estadual de Londrina e todos os idosos que aceitaram participar da pesquisa foram esclarecidos de seus objetivos e direitos, assinando termo de consentimento livre e esclarecido.

\section{Resultados}

Foram estudados 267 idosos de 60 a 74 anos, com idade média de 66,5 anos e predomínio do sexo feminino - 215 mulheres (80,5\%). O total de perdas foi de 69 indivíduos (20,5\%), assim representadas: recusa em participar - 13 (18,8\%), alteração de endereço - $13(18,8 \%)$ e 43 indivíduos não foram encontrados em suas residências, após três tentativas em dias e horários diversos $(62,3 \%)$.

\begin{tabular}{|c|c|c|}
\hline \multicolumn{3}{|c|}{ Tabela 2 - Drogas psicoativas utilizadas } \\
\hline Drogas utilizadas & $\mathbf{N}=3 I^{*}$ & $\%$ \\
\hline Amitriptilina & 8 & 25,8 \\
\hline Clonazepan & 6 & 19,4 \\
\hline Diazepan & 4 & 12,9 \\
\hline Alprazolan & 3 & 9,7 \\
\hline Sertralina & 3 & 9,7 \\
\hline Fluoxetina & 2 & 6,5 \\
\hline Bromazepan & 2 & 6,5 \\
\hline Carbamazepina & 2 & 6,5 \\
\hline Imipramina & । & 3,2 \\
\hline Flurazepan & । & 3,2 \\
\hline Lorazepan & I & 3,2 \\
\hline Clorpropamina & । & 3,2 \\
\hline Fenitoína & । & 3,2 \\
\hline Zolpiden & । & 3,2 \\
\hline Flunitrazepan & । & 3,2 \\
\hline
\end{tabular}

* Alguns idosos usavam mais do que um medicamento

\begin{tabular}{|c|c|c|c|}
\hline \multicolumn{4}{|c|}{$\begin{array}{l}\text { Tabela 3 - Análise multivariada da relação entre fluxo salivar baixo e o uso de } \\
\text { drogas psicoativas em idosos, ajustada para idade, sexo e o tabagismo }\end{array}$} \\
\hline Variáveis independentes & OR & IC 95\% & Valor do $\mathrm{p}$ \\
\hline $\begin{array}{l}\text { Uso de drogas psicoativas } \\
\text { Sexo } \\
\text { Idade (em anos) } \\
\text { Tabagismo }\end{array}$ & $\begin{array}{l}\overline{2,25} \\
0,93 \\
0,99 \\
0,71\end{array}$ & $\begin{array}{l}1,03-4,9 \mid \\
0,47-1,82 \\
0,93-1,06 \\
0,33-1,53\end{array}$ & $\begin{array}{l}0,04 \\
0,84 \\
0,98 \\
0,39\end{array}$ \\
\hline
\end{tabular}

Apenas 21,6\% dos indivíduos analisados freqüentaram escolas por pelo menos quatro anos, e 48,3\% foram classificados como integrantes das classes econômicas D e E (Tabela I).

$O$ uso de drogas psicoativas foi observado em 31 idosos ( $11,6 \%)$, com predomínio do uso de drogas benzodiazepínicos - 17 $(54,9 \%)$, seguidas dos antidepressivos tricíclicos - 10 (32,3\%) e dos inibidores da recaptação de serotonina - 5 (16,1\%) (Tabela 2).

A medida do fluxo salivar médio entre as mulheres foi de 0,74 $\mathrm{ml} / \mathrm{min}$ e entre os homens $0,84 \mathrm{ml} / \mathrm{min}$. Os valores que determinaram a distribuição dos tercis do fluxo salivar foram: primeiro tercil - 0,0 a 0,43 $\mathrm{ml} / \mathrm{min}$; segundo tercil - 0,44 a 0,76 ml/min; e terceiro tercil - 0,77 a 3,2 $\mathrm{ml} / \mathrm{min}$. Entre os usuários de drogas psicoativas, o fluxo salivar médio foi de $0,67 \mathrm{ml} / \mathrm{min}$ (desvio padrão=0,53) e nos demais foi de $0,78 \mathrm{ml} / \mathrm{min}$ (desvio padrão $=0,55)(p=0,2 \mathrm{l}$ ).

$\mathrm{Na}$ análise multivariada, os dados mostram uma associação do fluxo salivar abaixo de 0,44 $\mathrm{ml} / \mathrm{min}$ (primeiro tercil) com o uso de drogas psicoativas $(O R=2,25 ; \quad I C$ 95\%: I,03-4,9l; $p=0,04)$, independentemente do sexo, da idade e da presença de tabagismo (Tabela 3).

\section{Discussão}

Os resultados mostram uma associação entre o uso de medicamentos psicoativos e o baixo fluxo salivar nesta população de idosos. 
Cabrera MAS ET AL.

É importante ressaltar que o estudo de idosos da comunidade não portadores de dependência funcional possibilita uma análise com menor interferência das comorbidades e facilita a extrapolação dos achados para outros segmentos de idosos da comunidade, com características semelhantes.

Os dados identificam uma baixa utilização de medicamentos psicoativos neste grupo de idosos $(11,6 \%)$, quando comparada a outros autores brasileiros que detectaram prevalências de $13,5 \%{ }^{18}$ e de $22 \%{ }^{19}$. No entanto, apesar de pouco utilizadas, há um predomínio de utilização de drogas consideradas inadequadas a pacientes geriátricos, como os benzodiazepínicos e os antidepressivos tricíclicos ${ }^{20}$. Esse perfil de utilização de medicamentos psicoativos pode ser justificado pelo baixo poder aquisitivo da população estudada, com acesso apenas às drogas disponíveis pelo serviço público de saúde.

O fluxo salivar medido foi menor nas mulheres que nos homens. Este fato também foi identificado por outros autores, em populações de idosos ${ }^{11,13,14,21-23}$. No entanto, essa diferente apresentação do fluxo salivar entre homens e mulheres ainda não foi completamente investigada, mas pode estar contribuindo para as melhores condições de saúde bucal observada entre os idosos do sexo masculino ${ }^{24,25}$.

Os resultados obtidos confirmaram a possibilidade de menor fluxo salivar nos idosos usuários de drogas psicoativas, mesmo considerando a interferência de importantes variáveis confundidoras, como a idade, sexo e o tabagismo.

Thomson et. al. ${ }^{14}$ também analisaram um grupo de idosos da comunidade em um estudo prospectivo e identificaram o uso de polifarmácia e de antidepressivos como preditores de baixo fluxo salivar. Outros autores identificaram esta associação, mas não especificamente com drogas psicoativas ${ }^{13,15}$.

Diferentemente do presente estudo, essas poucas análises existentes não avaliaram a interferência do tabagismo, que pode estar associado a um aumento de fluxo salivar ${ }^{14}$. Além disso, estudaram todos os idosos da comunidade, incluindo os com alto grau de dependência funcional.

O volume do fluxo salivar médio observado nesses idosos usuários de drogas psicoativas $(0,67 \mathrm{ml} / \mathrm{min})$ foi inferior aos valores considerados como adequados para idosos $-0,70 \mathrm{ml} / \mathrm{min}^{10}$. Esta baixa do fluxo salivar, independente da queixa de xerostomia, é um importante determinante de alterações nutricionais nessa população de idosos ${ }^{4,23,26}$

Algumas limitações deste estudo merecem ser ressaltadas, como a elevada percentagem de perdas e a baixa proporção de homens na amostra analisada, decorrentes do grande número de idosos do sexo masculino que não se encontravam em seu domićlio durante a visita.

\section{CONCLUSÃo}

Os resultados obtidos mostram que, nesta população de idosos independentes e não institucionalizados, há uma diminuição do fluxo salivar nos indivíduos que usavam continuamente algum tipo de droga psicoativa.
Estas conclusões fortalecem a idéia de se evitar o uso indiscriminado de drogas psicoativas em indivíduos idosos. Além dos possíveis efeitos indesejáveis em relação ao sistema nervoso e cardiovascular, podem comprometer a saúde bucal e as condições nutricionais, que são muito importantes na promoção de saúde desta faixa etária.

\section{Conflito de interesse: não há.}

\section{SUMMARY}

\section{SALIVARY FLOW AND PSYCHOACTIVE DRUG CONSUMPTION IN ELDERLY} PEOPLE

OBJECTIVE. To analyze the association between low saliva flow rates and the use of psychoactive drugs among the elderly.

Methods. A cross-sectional study was carried out with 267 elderly people from 60 to 74 years of age who lived in a borough of the city of Londrina, Paraná State, Brazil. Individuals with high functional dependence or restricted to bed were excluded. Saliva flow rate was the dependent variable with values under the first tercile being considered as low flow rates (less than $0.44 \mathrm{ml} / \mathrm{min}$ ). The continuous use of psychoactive drugs (antidepressant, antiepileptic, sedative, antipsychotic, hypnotic or sedative-hypnotic drugs) was the independent variable. Multivariate analysis was performed taking into account gender, age and smoking status.

RESULTS. The majority of the elderly were women (80.5\%), with a mean age of 66.5 years. Use of psychoactive drugs was observed among 31 elderly (11.6\%). Mean saliva flow rate was $0.76 \mathrm{ml} / \mathrm{min}$, lower among users of psychoactive drugs $(0.67 \mathrm{~m} / / \mathrm{min})$. In the multivariate analysis, use of psychoactive drugs was associated with low saliva flow rates $(<0.44 \mathrm{ml} / \mathrm{min})$, independent of gender, age or smoking.

CONCLUSION. Results show that there is an association between use of psychoactive drugs and low saliva flow rates in this group of independent and non-institutionalized elderly. These conclusions stress the need of a rational use of these drugs, particularly among the elderly. [Rev Assoc Med Bras 2007; 53(2): 178-81]

KEY WORDS: Saliva. Xerostomia. Drug utilization. Aged.

\section{REFERÊNCIAS}

I. Giron MST, Fastbom J. Winblad B. Clinical trials of potential antidepressants: to what extent are the elderly represented: a review. Int J Geriatr Psychiatry. 2005;20(3):201-17.

2. Lechevallier N, Fourrier A, Berr C. Benzodiazepine use in the elderly: the EVA Study. Rev Epidemiol Sante Publique. 2003;5I(3):317-26.

3. Souza JC, Magna LA. Sonolência excessiva diurna e uso de hipnóticos em idosos, Rev Psiquiatr Clín. 2003;30(3):80-85.

4. Ship JA, Pillemer SR, Baum BJ. Xerostomia and the geriatric patient. J Am Geriatric Soc. 2002;50(3):535-43.

5. Scully CCBE. Drug effects on salivary glands: dry mouth. Oral Dis.2003; 9(4): 165-76

6. Pedersen AM. Saliva and gastrointestinal functions of taste, mastication, swallowing and digestion. Oral Dis. 2002;8(3): I17-29.

7. Torres SVS. Saúde Bucal: alterações fisiológicas e patológicas do envelhecimento. In: Freitas EV, Py L, Néri AL, Cançado FAX, Gorzoni ML, Rocha SM. Tratado de geriatria e gerontologia. Rio de Janeiro: 
Editora Guanabara Koogan; 2002. p.883.

8. Atkinson JC, Grisius M, Massey W. Salivary hypofunction and xerostomia: diagnosis and treatment. Dent Clin North Am. 2005;49(2):309-26.

9. Castro SAFN, Hartmann ACVC, Santos AC. Medicamentos associados à xerostomia e a distúrbios das funções orofaciais em idosos. Rev Fonoaudiol Bras. 2004;5(1):1-3.

10. Lima AAS, Machado DFM, Santos AW, Grégio AMT. Avaliação sialométrica em indivíduos da terceira idade. Rev Odonto Ciênc. 2004; I 9(45):238-44.

I I. Field EA, Fear S, Higham SM, Ireland RS, Rostron J, Willetts RM, et al. Age and medication are significant risk factors for xerostomia in an English population, attending general dental practice. Gerodontology. 200|: | 8(1):21-4.

12. Nagler RM. Salivary glands and the aging process: mechanistic aspects, health-status and medicinal-efficacy monitoring. Biogerontology. 2004:5(4):223-33.

1 3. Maciel SML. Idoso: uso de medicaçäo e seu reflexos na saúde bucal. Pesq Bras Odontoped Clin. Integr. 2002;2:89-95.

14. Thomson WM, Chalmers JM, Spencer AJ, Slade GD. Medication and dry mouth: findings from a cohort study of older people. J Public Health Dent. 2000;60(I): 12-20.

15. Nagler RM, Herskovich O. Relationships between age, drugs, oral sensorial complaints and salivary profile. Arch Oral Biol. 2005;50(1):7-16

16. ABEP. Associação Brasileira de Empresas de Pesquisa. CCEB: Critério de Classificação Econômica Brasil. [citado 2005 jul. 18]. Disponível em: http://www.anep.org.br/codigosguias/ABEP CCEB.pdf.

17. Decano AG. Epi Info 2002: um banco de dados e programa de estatística para profissionais de saúde pública para uso em Windows 95, 98, NT, e 2002. Atlanta: Centro para Controle e Prevenção de Doenças: 2002.

18. Coelho Filho JM, Marcopito LF, Castelo A. Perfil de medicamentos por idosos em área urbana do Nordeste do Brasil. Rev Saude Publica. 2004;38(4):557-64.

19. Flores LM, Mengue SS. Uso de medicamentos por idosos na região Sul do Brasil. Rev Saúde Pública. 2005; 39(6):924-9.
20. Beers $M H$. Explicit criteria for determining potentially inappropriate medication use by the elderly. An update. Arch Intern Med. | 997; | 57(| 4): | 53 |-6

21. Pajukoski H, Meurman J, Snellman-Grohn S, Keinanen S, Sulkava R. Salivary flow and composition in elderly patients referred to an acute care geriatric ward. Oral Surg, Oral Med Oral Pathol Oral Radiol Endod. 1997;84(3):265-7।.

22. Hochberg MC, Tielsch J, Munoz B, Bandeen-Roche K, West SK, Schein OD. Prevalence of symptoms of dry mouth and their relationship to saliva production in community dwelling elderly: the SEE project. Salisbury Eye Evaluation. J Rheumatol. |998;25(3):486-91

23. Ikebe K, Sajima H, Kobayashi S, Hata, K, Morii K, Nokubi T et al. Association of salivary flow rate with oral function in a sample of community-dwelling older adults in Japan. Oral Surg Oral Med Oral Pathol Oral Radiol Endod. 2002;94(2): I 84-90.

24. Colussi CF, Freitas SFT, Calvo MCM. Perfil epidemiológico da cárie e do uso e necessidade de prótese na população idosa de Biguaçu, Santa Catarina. Rev Bras Epidemiol. 2004;7:88-97.

25. Mesas AE. Condições de saúde bucal, estado nutricional e fatores associados em idosos de Londrina [dissertação]. Londrina: Universidade Estadual de Londrina; 2005.

26. Loesche W], Bretz WA, Grossman NS, Lopatin DE. Dental findings in geriatric populations with diverse medical backgrounds. Oral Surg Oral Med Oral Pathol Oral Radiol Endodm 1995;80(I):43-54.

Artigo recebido: 02/10/06 Aceito para publicação: 29/10/06 